\title{
Cospectral Bipartite Graphs with the Same Degree Sequences but with Different Number of Large Cycles
}

\author{
Ali Dehghan and Amir H. Banihashemi \\ Department of Systems and Computer Engineering, Carleton University, Ottawa, Ontario, Canada
}

\begin{abstract}
Finding the multiplicity of cycles in bipartite graphs is a fundamental problem of interest in many fields including the analysis and design of low-density parity-check (LDPC) codes. Recently, Blake and Lin computed the number of shortest cycles ( $g$-cycles, where $g$ is the girth of the graph) in a bi-regular bipartite graph, in terms of the degree sequences and the spectrum (eigenvalues of the adjacency matrix) of the graph [IEEE Trans. Inform. Theory 64(10):6526-6535, 2018]. This result was subsequently extended in [IEEE Trans. Inform. Theory, accepted for publication, Dec. 2018] to cycles of length $g+2, \ldots, 2 g-2$, in bi-regular bipartite graphs, as well as 4-cycles and 6-cycles in irregular and halfregular bipartite graphs, with $g \geq 4$ and $g \geq 6$, respectively. In this paper, we complement these positive results with negative results demonstrating that the information of the degree sequences and the spectrum of a bipartite graph is, in general, insufficient to count (a) the $i$-cycles, $i \geq 2 g$, in bi-regular graphs, (b) the $i$-cycles for any $i>g$, regardless of the value of $g$, and $g$-cycles for $g \geq 6$, in irregular graphs, and (c) the $i$-cycles for any $i>g$, regardless of the value of $g$, and $g$-cycles for $g \geq 8$, in half-regular graphs. To obtain these results, we construct counter-examples using the Godsil-McKay switching.

Index Terms: Cycle multiplicity, bipartite graphs, Tanner graphs, graph spectrum, low-density parity-check (LDPC) codes, bi-regular bipartite graphs, irregular bipartite graphs, half-regular bipartite graphs, girth.
\end{abstract}

\section{INTRODUCTION}

Bipartite graphs are commonly used in science and engineering to represent systems, where the nodes on one side of the bipartition represent the variables, and the nodes on the other side represent local constraints, each involving its adjacent variables, see, e.g., [20]. A well-known example is the Tanner graph representation [30] of low-density parity-check (LDPC) codes [8].

This paper was presented in part at ISTC 2018, Hong Kong. This research was supported by NSERC Discovery Grant 217239-2013-RGPIN. 
In graph representations, cycles and degree sequences often play an important role in determining the performance of the system. For example, in the case of LDPC codes, the performance of iterative message-passing algorithms, that are used in practice for the decoding, depends highly on the cycle distribution and the degree sequences of the underlying Tanner graph [1], [11], [12], [14], [16]-[18], [27], [28], [32]. For the purpose of analysis and design of systems and codes, it is thus important to know the number of cycles of different length in the corresponding bipartite graphs, and the relationships that may exist between the cycle distribution and the degree sequences of the graph.

The connection between the performance of LDPC codes and cycles of the Tanner graph has motivated much research on the study of the cycle distribution and the counting of cycles in bipartite graphs, see, e.g., [2], [6], [7], [10], [19]. Counting cycles of a given length, even in bipartite graphs, is known to be NP-hard [24]. In [19], Karimi and Banihashemi presented an efficient message-passing algorithm to count the number of cycles of length less than $2 g$, in a general graph, where $g$ is the girth of the graph. The distribution of cycles in different ensembles of bipartite graphs was studied in [6], where it was shown that for random ensembles of bipartite graphs, the multiplicities of cycles of different lengths have independent Poisson distributions with the expected values only a function of the cycle length and the degree distribution (and independent of the size of the graph). More recently, Blake and Lin [2] presented a formula to compute the multiplicity of cycles of length $g$ in bi-regular bipartite graphs as a function of the spectrum (eigenvalues of the adjacency matrix of the graph) and degree sequences of the graph. This result was subsequently extended in [7] to compute the number of cycles of length $g+2, \ldots, 2 g-2$, in bi-regular bipartite graphs, as well as the number of 4-cycles and 6-cycles in irregular and half-regular bipartite graphs, with $g \geq 4$ and $g \geq 6$, respectively. It is noteworthy that, while the majority of techniques developed in the literature for counting cycles are algorithmic, the results in [2] and [7] are presented as closed-form formulas.

In relation to the results of [2] and [7], that use the spectrum $\left\{\lambda_{i}\right\}$ of a bipartite graph as part of the required information to derive the cycle multiplicities, we note that, in general, determining the properties of a graph from its spectrum is an active area of research in graph theory. For some examples, see [5], [22], [23], [26], [29]. It is known that $\sum_{i} \lambda_{i}^{j}$ for $j=1,2$, and 3 is equal to 0 , the number of edges in the graph, and six times the number of 3-cycles of the graph, respectively. It is, however, not possible to extend the last result to cycles of length larger than three. (For example, the complete bipartite graph with one and four nodes on the two sides of 
the bipartition, and the union of a 4-cycle and a single node are two bipartite graphs with the same spectrum $\{-2,0,0,0,2\}$, but with different number of 4-cycles.) The results of [2] and [7] make this extension possible but with the extra information about the degree sequences of the graph.

In this paper, we complement the results of [2] and [7] by demonstrating, through counterexamples, that the information of the degree sequences and the spectrum of a bipartite graph is, in general, insufficient to count (a) the $i$-cycles with $i \geq 2 g$ in bi-regular graphs, (b) the $i$-cycles for any $i>g$, regardless of the value of $g$, and $g$-cycles for $g \geq 6$, in irregular graphs, and (c) the $i$-cycles for any $i>g$, regardless of the value of $g$, and $g$-cycles for $g \geq 8$, in half-regular graphs. To construct our counter-examples, we use the Godsil-McKay switching [9], and prove that the application of such switches to bi-regular bipartite graphs preserves the degree sequences and the spectrum of the graph.

We note that in graph theory, the Godsil-McKay switching is a well-known tool to construct cospectral graphs. For example, Blázsik et al. [3] used the switching to construct two cospectral regular graphs such that one has a perfect matching while the other does not have any perfect matching. For more applications, see [15], [21], [25].

The summary of the results regarding the possibility of computing the number of cycles of different length in different types of bipartite graphs with different girth using only the spectrum and the degree sequences of the graph is presented in Table I. In this table, the notation "P" ("IP") is used to mean that it is possible (impossible), in general, to find the multiplicity of cycles of a given length in a graph from the spectrum and the degree sequences of the graph.

The organization of the rest of the paper is as follows: In Section II, we present some definitions and notations. Next, in Section III, we construct two bi-regular bipartite graphs such that they have the same spectrum, degree sequences and girth, but different number of $i$-cycles for $i \geq 2 g$. This demonstrates that, in general, it is not possible to determine the number of $i$-cycles for $i \geq 2 g$ in a bi-regular bipartite graph as a function of only the spectrum and the degree sequences of the graph. In Section IV, we study the possibility of computing the multiplicity of short cycles of irregular bipartite graphs using only the spectrum and degree sequences, and demonstrate through some graph constructions that the answer is generally negative, except for the case of 4-cycles in graphs with $g \geq 4$ (the equation for the multiplicity of 4-cycles was derived in [7] as a function of graph spectrum and its degree sequences). In Section V, we continue our study of computing the multiplicity of short cycles in half-regular bipartite graphs, and show that for 
TABLE I

A SUMMARY OF THE RESULTS ON THE POSSIBILITY OF COUNTING CYCLES OF LENGTH $i$ IN BI-REGULAR, HALF-REGULAR AND IRREGULAR BIPARTITE GRAPHS WITH GIRTH $g$ USING ONLY THE SPECTRUM AND THE DEGREE SEQUENCES OF THE GRAPH. (NOTATIONS “P” AND “IP” ARE USED FOR “POSSIBLE” AND “IMPOSSIBLE,” RESPECTIVELY.)

\begin{tabular}{|c|c|c|c|c|}
\hline & & $i=g$ & $g+2 \leq i \leq 2 g-2$ & $2 g \leq i$ \\
\hline Bi-regular & $g \geq 4$ & $\mathrm{P}[2]$ & $P$ [7] & IP (Section 【) \\
\hline \multirow{3}{*}{ Half-regular } & $g=4$ & $\mathrm{P}[7$ & IP (Subsection IV-A & IP (Subsection IV-A \\
\hline & $g=6$ & P [7] & IP (Section V) & IP (Section \) \\
\hline & $g \geq 8$ & IP (Section V) & IP (Section V) & IP (Section V) \\
\hline \multirow{3}{*}{ Irregular } & $g=4$ & P [7] & IP (Subsection IV-A & IP (Subsection IV-A \\
\hline & $g=6$ & IP (Subsection IV-C) & IP (Subsection IV-C) & IP (Subsection IV-C) \\
\hline & $g \geq 8$ & IP (Subsection IV-B & IP (Subsection IV-B) & IP (Subsection IV-B \\
\hline
\end{tabular}

all girths and cycle sizes, with the exception of 6-cycles in graphs with $g \geq 6$ (and 4-cycles in graphs with $g \geq 4$ ), the information of only the spectrum and degree sequences is insufficient to count the cycles. The paper is concluded with some remarks in Section VI,

\section{DEFINITIONS AND NOTATIONS}

A graph $G$ is defined as a set of vertices or nodes $V(G)$ and a set of edges $E(G)$, where $E(G)$ is a subset of the pairs $\{\{v, u\}: v, u \in V(G), v \neq u\}$. The shorthands $V$ and $E$ are used if there is no ambiguity about the graph. An edge $e \in E$ with endpoints $u \in V$ and $w \in V$ is denoted by $\{u, w\}$, or by $u w$ or $w u$, in brief. Throughout this work, we consider undirected graphs with no loop or parallel edges (i.e., simple graphs).

A walk of length $k$ in the graph $G$ is a sequence of nodes $v_{1}, v_{2}, \ldots, v_{k+1}$ in $V$ such that $\left\{v_{i}, v_{i+1}\right\} \in E$, for all $i \in\{1, \ldots, k\}$. A walk is a path if all the nodes $v_{1}, v_{2}, \ldots, v_{k}$ are distinct. A walk is called a closed walk if the two end nodes are identical, i.e., if $v_{1}=v_{k+1}$. Under the same condition, a path is called a cycle.

The length of a walk, path or cycle is the number of its edges. We use the notation $P_{n}$ to denote a path with $n$ nodes. We denote cycles of length $k$, also referred to as $k$-cycles, by $C_{k}$. The length of the shortest cycle(s) in a graph is called girth and is denoted by $g$.

A graph $G=(V, E)$ is called bipartite, if the node set $V$ can be partitioned into two disjoint subsets $U$ and $W$, i.e., $V=U \cup W$ and $U \cap W=\emptyset$, such that every edge in $E$ connects a 
node from $U$ to a node from $W$. A graph is bipartite if and only if the lengths of all its cycles are even. Tanner graphs of LDPC codes are bipartite graphs, in which $U$ and $W$ are referred to as variable nodes and check nodes, respectively. Parameters $n$ and $m$ in this case are used to denote $|U|$ and $|W|$, respectively. Parameter $n$ is the code's block length and the code rate $R$ satisfies $R \geq 1-(m / n)$.

A graph is called complete if every node is connected to all the other nodes. We use the notation $K_{a}$ for a complete graph with a nodes. A bipartite graph $G(U \cup W, E)$ is called complete, and is denoted by $K_{|U|,|W|}$, if every node in $U$ is connected to every node in $W$.

The number of edges incident to a node $v$ is called the degree of $v$, and is denoted by $d(v)$. The degree sequences of a bipartite graph $G$ are defined as the two monotonic nonincreasing sequences of the node degrees on the two sides of the graph. For example, the complete bipartite graph $K_{4,3}$ has degree sequences $(3,3,3,3)$ and $(4,4,4)$. The degree sequences also contain the information about the number of nodes on each side of the graph. A bipartite graph $G=(U \cup W, E)$ is called bi-regular, if all the nodes on the same side of the bipartition have the same degree, i.e., if all the nodes in $U$ have the same degree $d_{u}$ and all the nodes in $W$ have the same degree $d_{w}$. We also call such graphs $\left(d_{u}, d_{w}\right)$-regular graphs. It is clear that, for a bi-regular graph, $|U| d_{u}=|W| d_{w}=|E(G)|$. A bipartite graph is called half-regular, if all the nodes on one side of the bipartition have the same degree. A half-regular Tanner graph can be either variable-regular or check-regular. A bipartite graph that is not bi-regular is called irregular. With this definition, half-regular graphs are a special case of irregular graphs.

A graph $G$ is connected, if there is a path between any two nodes of $G$. If the graph $G$ is not connected, we say that it is disconnected. A connected component of a graph is a connected subgraph such that there are no edges between nodes of the subgraph and nodes of the rest of the graph.

The adjacency matrix of a graph $G$ is the matrix $A=\left[a_{i j}\right]$, where $a_{i j}$ is the number of edges connecting the node $i$ to the node $j$ for all $i, j \in V$. The matrix $A$ is symmetric and since we have assumed that $G$ has no parallel edges or loops, $a_{i j} \in\{0,1\}$, for all $i, j \in V$, and $a_{i i}=0$, for all $i \in V$. The set of the eigenvalues $\left\{\lambda_{i}\right\}$ of $A$ is called the spectrum of the graph. It is well-known that the spectrum of a disconnected graph is the disjoint union of the spectra of its components [31]. One important property of the adjacency matrix is that the number of walks between any two nodes of the graph can be determined using the powers of this matrix. More precisely, the entry in the $i^{\text {th }}$ row and the $j^{\text {th }}$ column of $A^{k},\left[A^{k}\right]_{i j}$, is the number of walks of 
length $k$ between nodes $i$ and $j$. In particular, $\left[A^{k}\right]_{i i}$ is the number of closed walks of length $k$ containing node $i$. The total number of closed walks of length $k$ in $G$ is thus $\operatorname{tr}\left(A^{k}\right)$, where $\operatorname{tr}(\cdot)$ is the trace of a matrix. Since $\operatorname{tr}\left(A^{k}\right)=\sum_{i=1}^{|V|} \lambda_{i}^{k}$, it follows that the multiplicity of closed walks of different length in a graph can be obtained using the spectrum of the graph. It is also known that $\sum_{i} \lambda_{i}(G)^{j}$, for $j=1,2,3$, is equal to $0,|E(G)|$, and $6 \times N_{3}(G)$, respectively, where $N_{3}(G)$ is the number of 3-cycles in $G$. It is, however, not possible to extend the last result to cycles of length larger than three, and find the multiplicity of such cycles as a function of only the spectrum. For example, the complete bipartite graph $K_{1,4}$ and the graph $C_{4} \cup K_{1}$ (the union of a 4 -cycle and a single node) are two bipartite graphs with the same spectrum $\{-2,0,0,0,2\}$, but with different number of 4 -cycles.

To devise our counter-examples, we often use cycles and paths. The path graph $P_{n}$ has the following spectrum:

$$
2 \cos \left(\frac{\pi j}{n+1}\right), j=1, \ldots, n,
$$

and the spectrum of a cycle of length $n, C_{n}$, is as follows:

$$
2 \cos \left(\frac{2 \pi j}{n}\right), j=0, \ldots, n-1 .
$$

In general, the spectrum of a graph does not uniquely determine the graph. Two graphs are called cospectral or isospectral if they have the same spectrum. On the other hand, there are graphs that are known to be uniquely determined by their spectrum. Two examples are the complete graph $K_{n}$, and the complete bipartite graph $K_{n, n}$ [31].

\section{COUNTING LARGE CYCLES IN BI-REGULAR BIPARTITE GRAPHS}

In this section, we demonstrate that the knowledge of spectrum and degree sequences of a biregular bipartite graph is not in general sufficient to determine the multiplicity of cycles of length $2 g$ and larger. We start by providing a counter-example of two regular bipartite graphs whose spectrum, degree sequences and girth are identical but have different number of cycles of length $2 g$ and larger. To construct this counter-example, we use the concept of switching in graphs and in particular, Godsil-McKay switching [9]. The latter is a graph transformation that maintains the spectrum of the graph. We also prove that Godsil-McKay switching, in general, maintains the degree sequences of a bi-regular bipartite graph and can thus be used to construct cospectral bi-regular bipartite graphs with similar degree sequences, but different cycle distributions for cycle lengths larger than $2 g-2$. 
Theorem 1. [Godsil-McKay switching [9]] Let $G$ be a graph and let $\left\{X_{1}, \ldots, X_{\ell}, Y\right\}$ be a partition of the node set $V(G)$ of $G$. Suppose that for every node $y \in Y$, and every $i \in$ $\{1, \ldots, \ell\}$, the node $y$ has either $0, \frac{1}{2}\left|X_{i}\right|$ or $\left|X_{i}\right|$ neighbors in $X_{i}$. Moreover, suppose that for each $i, j \in\{1, \ldots, \ell\}$ ( $i$ and $j$ can be equal), all the nodes in $X_{i}$ have the same number of neighbors in $X_{j}$. Construct a new graph $G^{\prime}$ as follows: For each $y \in Y$ and $i \in\{1, \ldots, \ell\}$ such that $y$ has $\frac{1}{2}\left|X_{i}\right|$ neighbors in $X_{i}$, delete the corresponding $\frac{1}{2}\left|X_{i}\right|$ edges and join $y$ instead to the $\frac{1}{2}\left|X_{i}\right|$ other nodes in $X_{i}$. Then, the graphs $G$ and $G^{\prime}$ are cospectral.

In the above process, the node partition $\left\{X_{1}, \ldots, X_{\ell}, Y\right\}$ is called a Godsil-McKay switching partition.

In the following, we construct a 3-regular bipartite graph $G$ with girth six. We then use Theorem 1 to convert $G$ to $G^{\prime}$, such that $G^{\prime}$ is also 3-regular and bipartite, and $G$ and $G^{\prime}$ are cospectral. In our construction of $G$, we use the Heawood graph [4], shown in Fig. 1. (In the rest of the paper, to make the identification of the nodes on each side of the bipartition easier, we sometimes use black and white colors to distinguish them.) The Heawood graph is a 3-regular bipartite graph with girth six.

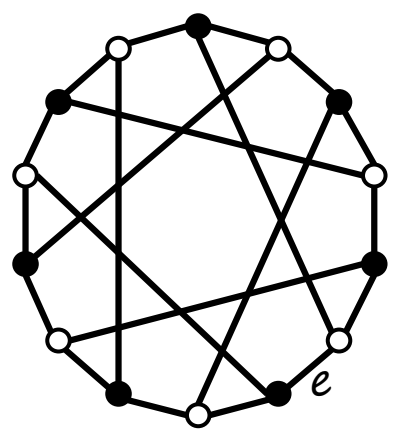

Fig. 1. The Heawood graph.

Construction of $G$ : Consider two disjoint cycles of length 6 and 18 with nodes $d_{1}, d_{2}, \ldots, d_{6}$, and $a_{1}, a_{2}, \ldots, a_{6}, b_{1}, \ldots, b_{6}, c_{1}, \ldots, c_{6}$, respectively. Add to the graph twelve nodes $v_{1}, u_{1}, \ldots, v_{6}, u_{6}$, and for each $i \in\{1,2, \ldots, 6\}$, add the edges $v_{i} d_{i}, v_{i} a_{i}, u_{i} b_{i}, u_{i} c_{i}$. Further add to the graph nodes $v^{\prime}, v^{\prime \prime}, u^{\prime}, u^{\prime \prime}$, and edges $v^{\prime} v_{1}, v^{\prime} v_{3}, v^{\prime} v_{5}, v^{\prime \prime} v_{2}, v^{\prime \prime} v_{4}, v^{\prime \prime} v_{6}, u^{\prime} u_{3}, u^{\prime} u_{5}, u^{\prime \prime} u_{4}, u^{\prime \prime} u_{6}, u^{\prime} u^{\prime \prime}$. Now, add a copy of the Heawood graph, and remove one of its circumferential edges such as $e=z z^{\prime}$ (see Fig. 1). Finally, add the edges $u_{1} z$ and $u_{2} z^{\prime}$ to the graph. The resulting graph is a 3-regular bipartite graph with girth six. We call this graph $G$. See Fig. 2. 


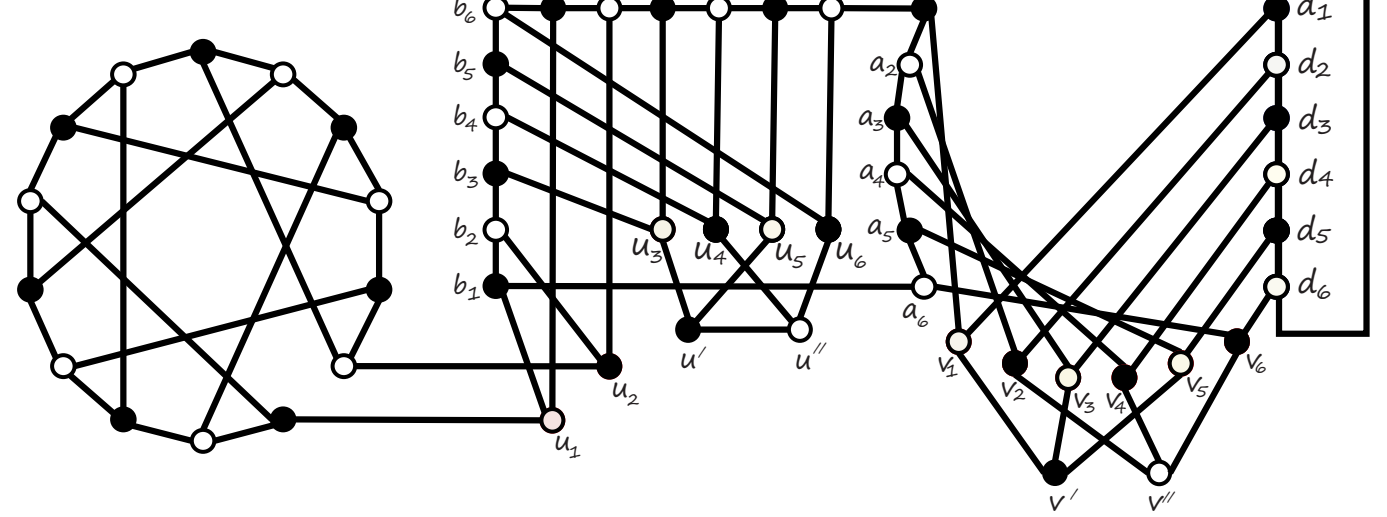

Fig. 2. The 3-regular bipartite graph $G$.

Construction of $G^{\prime}$ : We use Theorem 1, and construct $G^{\prime}$ from $G$. Let $\ell=6$, and for each $i, 1 \leq i \leq 6$, let $X_{i}=\left\{a_{i}, b_{i}, c_{i}, d_{i}\right\}$. Also, Let $Y=V(G) \backslash \cup_{i=1}^{6} X_{i}$. It can be seen that for every node $y \in Y$, and every $i \in\{1, \ldots, 6\}$, node $y$ has either 0 or 2 neighbors in $X_{i}$ (note that for each $\left.i,\left|X_{i}\right|=4\right)$. Also, for each pair $i, j \in\{1, \ldots, \ell\}$, all the nodes in $X_{i}$ have the same number of neighbors in $X_{j}$ (see Table II). Consequently, the partitioning has all the properties of Theorem 1. We can thus apply Godsil-McKay switching, and obtain $G^{\prime}$. (See Fig. 3.)

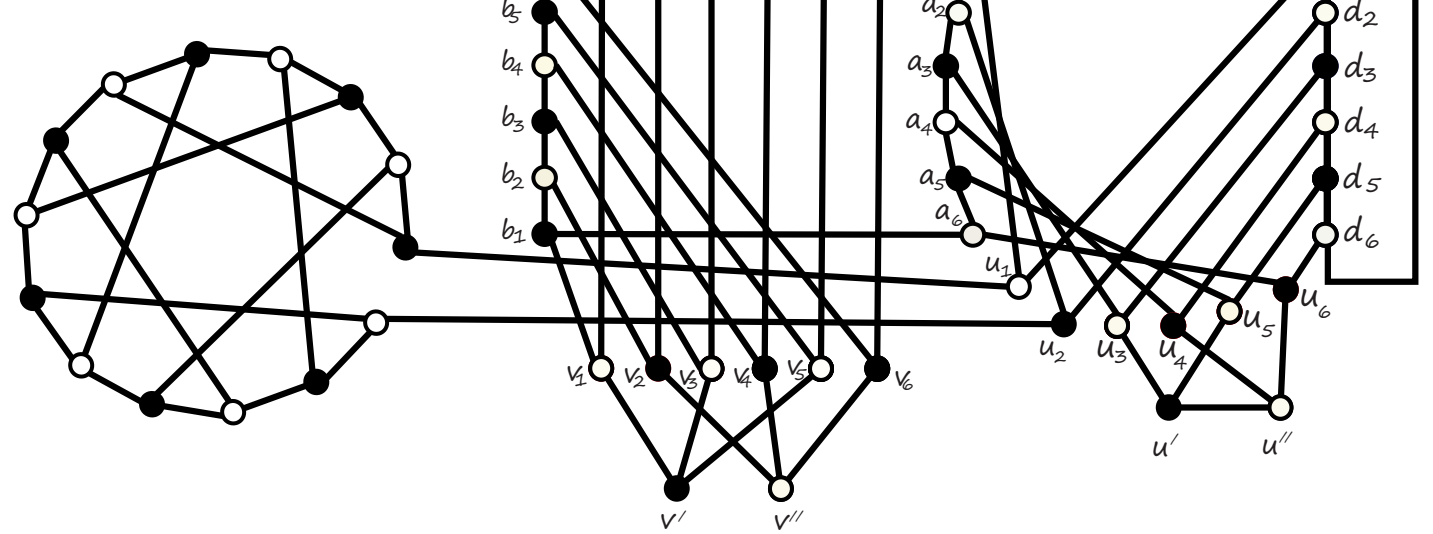

Fig. 3. The 3-regular bipartite graph $G^{\prime}$, obtained by the application of Godsil-McKay switching to the graph $G$ in Fig. 2

Both $G$ and $G^{\prime}$ are 3-regular bipartite graphs and based on Theorem 1, both have the same 
TABLE II

THE $(i, j)$ ENTRY OF THE TABLE SHOWS THE NUMBER OF NEIGHBORS THAT AN ARBITRARY NODE $v \in X_{i}$ HAS IN THE SET $X_{j}$ (FOR GODSIL-MCKAY SWITCHING PARTITION OF $G$ SHOWN IN FIG. 2).

\begin{tabular}{|c||c|c|c|c|c|c|}
\hline & $X_{1}$ & $X_{2}$ & $X_{3}$ & $X_{4}$ & $X_{5}$ & $X_{6}$ \\
\hline \hline$X_{1}$ & 0 & 1 & 0 & 0 & 0 & 1 \\
\hline$X_{2}$ & 1 & 0 & 1 & 0 & 0 & 0 \\
\hline$X_{3}$ & 0 & 1 & 0 & 1 & 0 & 0 \\
\hline$X_{4}$ & 0 & 0 & 1 & 0 & 1 & 0 \\
\hline$X_{5}$ & 0 & 0 & 0 & 1 & 0 & 1 \\
\hline$X_{6}$ & 1 & 0 & 0 & 0 & 1 & 0 \\
\hline
\end{tabular}

spectrum. In Table III, we have listed the number of cycles of length 6 up to 22, for both graphs 1 As expected from the results presented in [7], both graphs have the same cycle distribution for cycles of length up to $2 g-2=10$. From the table, however, it can be seen that the multiplicities of cycles of length $2 g=12$ and larger are different in these graphs.

TABLE III

MULTIPLICITIES OF CYCLES OF LENGTH 6 UP TO 22 IN $G$ AND $G^{\prime}$

\begin{tabular}{|c||c|c|c|c|c|c|c|c|c|}
\hline Graph & 6-cycles & 8-cycles & 10-cycles & 12-cycles & 14-cycles & 16-cycles & 18-cycles & 20-cycles & 22-cycles \\
\hline$G$ & 51 & 54 & 186 & 212 & 460 & 659 & 1609 & 4038 & 11132 \\
\hline$G^{\prime}$ & 51 & 54 & 186 & 213 & 458 & 669 & 1576 & 4090 & 10977 \\
\hline
\end{tabular}

Although, the example just provided was for regular bipartite graphs, one can use the following theorem to construct cospectral $\left(d_{u}, d_{w}\right)$-regular bipartite graphs with $d_{u} \neq d_{w}$, whose $i$-cycle multiplicities are different for $i \geq 2 g$.

Theorem 2. Let $G$ be a bi-regular bipartite graph, and suppose that Godsil-McKay switching is used to convert $G$ into $G^{\prime}$. Then, the graph $G^{\prime}$ is also bi-regular and both graphs have the same degree sequences.

\footnotetext{
${ }^{1}$ The cycles are counted using a Matlab program by Jeff Howbert [13]. This program counts all cycles in a simple undirected graph up to a specified size limit, using a backtracking algorithm.
} 
Proof. For the proof, we first discuss some of the properties of a Godsil-McKay switching partition of a bi-regular bipartite graph. Let $G=(U \cup W, E)$ be a bi-regular graph in which all the nodes in $U$ have the same degree $d_{u}$ and all the nodes in $W$ have the same degree $d_{w}$. Let $\left\{X_{1}, \ldots, X_{\ell}, Y\right\}$ be a Godsil-McKay switching partition for the nodes of $G$. For each $i$, we say that the set of nodes $X_{i}$ is of Type 1 (Type 2), if all nodes of $X_{i}$ are in $U(W)$. Otherwise, we say that $X_{i}$ is of Type 3 (if some nodes of $X_{i}$ are in $U$ and some others are in $W$ ). Let $X_{i}$ be a set of Type 3. Partition $X_{i}$ into two parts $X_{i}^{1}$ and $X_{i}^{2}$, where $X_{i}^{1}$ is the subset of nodes of $X_{i}$ that are in $U$, and thus $X_{i}^{2}$ contains the nodes of $X_{i}$ that are in $W$. Therefore, $\left|X_{i}\right|=\left|X_{i}^{1}\right|+\left|X_{i}^{2}\right|$. If $X_{i}$ is of Type 3, we say it is of Type 3.1, if $\left|X_{i}^{1}\right|=\left|X_{i}^{2}\right|$. Otherwise, we say that $X_{i}$ is of Type 3.2. We then have the following properties for partition sets of different types.

Lemma 1. Any Godsil-McKay switching partition $\left\{X_{1}, \ldots, X_{\ell}, Y\right\}$ of the nodes of a $\left(d_{u}, d_{w}\right)$ regular bipartite graph $G=(U \cup W, E)$ has the following properties:

P1. There is no connection (edge) between the nodes of a Type-3 set and the nodes of a Type-1 or Type-2 set.

P2. There is no connection between the nodes of a Type-3.1 set and the nodes of a Type-3.2 set.

P3. Let $X_{i}$ and $X_{j}$ be two sets of Type 3.2. Assume that the nodes in $X_{i}$ have at least one neighbor in $X_{j}$. Then, if $\left|X_{i}^{1}\right|>\left|X_{i}^{2}\right|$, we have $\left|X_{j}^{1}\right|<\left|X_{j}^{2}\right|$, and if $\left|X_{i}^{1}\right|<\left|X_{i}^{2}\right|$, we have $\left|X_{j}^{1}\right|>\left|X_{j}^{2}\right|$.

P4. Let $X_{i}$ be a set of Type 3. If a node $y \in Y$ has a neighbor in $X_{i}$, then $y$ is adjacent with $\frac{\left|X_{i}\right|}{2}$ nodes of $X_{i}^{1}$ or $y$ is adjacent with $\frac{\left|X_{i}\right|}{2}$ nodes of $X_{i}^{2}$ (y cannot have neighbors in both $X_{i}^{1}$ and $X_{i}^{2}$ ).

P5. For each $X_{i}$, each node in $X_{i}$ is connected to the same number of nodes in $\cup_{j=1}^{\ell} X_{j}$.

Proof. P1. Let $X_{i}$ be a set of Type 3 and $X_{j}$ be a set of Type 2 or Type 1 . Since the graph is bipartite, some of the nodes of $X_{i}$ cannot have any connection to the nodes of $X_{j}$. Moreover, all the nodes of $X_{i}$ must have the same number of neighbors in $X_{j}$. This number thus must be zero. P2. Let $X_{i}$ be a set of Type 3.2 and $X_{j}$ be a set of Type 3.1. Let $\left|E^{\prime}\right|$ be the number of edges between $X_{i}^{1}$ and $X_{j}^{2}$, and $\left|E^{\prime \prime}\right|$ be the number of edges between $X_{i}^{2}$ and $X_{j}^{1}$. Since $X_{j}$ is a set of Type 3.1, and every node in $X_{j}$ has the same number of neighbors in $X_{i}$, we have $\left|E^{\prime}\right|=\left|E^{\prime \prime}\right|$. On the other hand, since $X_{i}$ is a set of Type 3.2 and every node in $X_{i}$ has the 
same number of neighbors in $X_{j}$, we have $\left|E^{\prime}\right| \neq\left|E^{\prime \prime}\right|$, which is a contradiction. The proofs for P3-P5 are straightforward.

We now prove Theorem 2, Consider the application of the Godsil-McKay switching to convert a $\left(d_{u}, d_{w}\right)$-regular bipartite graph $G=(U \cup W, E)$ into the graph $G^{\prime}$. The nodes of $G^{\prime}$ can be partitioned into two sets $U^{\prime}$ and $W^{\prime}$ according to the following rules:

Rule 1. For each node $v \in Y$ in the graph $G$, assign the corresponding node $v$ in $G^{\prime}$ to $U^{\prime}\left(W^{\prime}\right)$ if $v$ in $G$ is in $U(W)$.

Rule 2. For each $i$, if $X_{i}$ is of Type 1 or Type 2, then for each node $v$ in $X_{i}$ in the graph $G$, assign the corresponding node $v$ in $G^{\prime}$ to $U^{\prime}\left(W^{\prime}\right)$ if $v$ in $G$ is in $U(W)$.

Rule 3. For each $i$, if $X_{i}$ is of Type 3, then for each node $v$ in $X_{i}$ in the graph $G$, assign the corresponding node $v$ in $G^{\prime}$ to $U^{\prime}\left(W^{\prime}\right)$ if $v$ in $G$ is in $W(U)$.

Now, we show that $G^{\prime}=\left(U^{\prime} \cup W^{\prime}, E^{\prime}\right)$ is a bi-regular graph in which all the nodes in $U^{\prime}$ have the same degree $d_{u}$ and all the nodes in $W^{\prime}$ have the same degree $d_{w}$. To show this, we examine the degrees of different partition sets $Y$ and $X_{i}$ 's. For the latter sets, the examination is based on the type of the set.

(i) Set $Y$ : It is clear that the Godsil-McKay switching does not change the degree of any node in $Y$, and by Rule 1 , those nodes in $Y$ with degree $d_{u}\left(d_{w}\right)$ are in $U^{\prime}\left(W^{\prime}\right)$.

(ii) Type-1 or Type- $2 X_{i}$ : Let $X_{i}$ be a set of Type 1 . If there is no node $y \in Y$ such that $y$ is adjacent with $\frac{\left|X_{i}\right|}{2}$ nodes of $X_{i}$, then the Godsil-McKay switching does not change the degree of any node in $X_{i}$. Now, assume that there is a node $y \in Y$ such that $y$ is adjacent to $\frac{\left|X_{i}\right|}{2}$ nodes of $X_{i}$. Let $Y_{i} \subset Y$ be a subset of nodes such that for each node $y \in Y_{i}$, the node $y$ is adjacent to $\frac{\left|X_{i}\right|}{2}$ nodes of $X_{i}$. Considering that all nodes in $X_{i}$ have the same degree $d_{u}$, by using P5, we conclude that all the nodes in $X_{i}$ have the same number of neighbors in $Y_{i}$. Call this number $\gamma$. By counting the number of edges $\eta$ between $X_{i}$ and $Y_{i}$, we find that $\eta=\gamma\left|X_{i}\right|$. On the other hand, $\eta=\left|Y_{i}\right|\left|X_{i}\right| / 2$. Thus, $\left|Y_{i}\right|=2 \gamma$. This implies that each node $v \in X_{i}$ is adjacent to half of the nodes in $Y_{i}$ ( $\gamma$ of them), and has no connection to the other half. Consequently, the Godsil-McKay switching does not change the degree of any node in $X_{i}$. This together with Rule 2 shows that each node in any Type- 1 set in $U^{\prime}$ has degree $d_{u}$. The proof for a Type- 2 set is similar.

(iii) Let $X_{i}$ be a set of Type 3.1. By $\mathrm{P} 1$ and $\mathrm{P} 2$, all the connections to $X_{i}$ are from $Y$ and Type-3.1 sets. Based on P4, after applying the Godsil-McKay switching, the degree of each node 
in $X_{i}^{1}$ will be $d_{w}$ and the degree of each node in $X_{i}^{2}$ will be $d_{u}$. Thus, by Rule 3 , and the fact that $\left|X_{i}^{1}\right|=\left|X_{i}^{2}\right|$, the degree sequence of the graph does not change after switching. Moreover, it is easy to see that after the application of the switching to $X_{i}$, the graph still remains bipartite.

(iv) Let $X_{i}$ be a set of Type 3.2. We consider two cases:

Case 1. Without loss of generality, assume that $d_{w}>d_{u}$. In this case, by P4, P5, and the condition $d_{w}>d_{u}$, there must be a node $y^{\prime} \in Y$ such that $y^{\prime}$ is adjacent with $\frac{\left|X_{i}\right|}{2}$ nodes of $X_{i}^{2}$. Thus, $\left|X_{i}^{2}\right| \geq \frac{\left|X_{i}\right|}{2}$. This together with the definition of Type 3.2 sets, i.e., $\left|X_{i}^{1}\right| \neq\left|X_{i}^{2}\right|$, result in

$$
\left|X_{i}^{1}\right|<\left|X_{i}^{2}\right| \text {. }
$$

Note that (3) is valid for any set $X_{i}$ of Type 3.2. On the other hand, the set $X_{i}^{1}$ contains at least one node $v$. By $\mathrm{P} 1$ and $\mathrm{P} 2$, none of the $d_{u}$ connections of $v$ can be to any node in Type 1, Type 2 or Type 3.1 sets. The connections cannot be to the nodes in $X_{i}^{2}$ either, because this implies, by the condition of Godsil-McKay partitioning, that every node in $X_{i}$ must also be connected to $d_{u}$ other nodes in $X_{i}$. This however, is not possible because it would imply that there must be $\left|X_{i}^{2}\right| \times d_{u}$ connections from $X_{i}^{2}$ to $X_{i}^{1}$, which, by (3), is more than the total number of edges connected to $X_{i}^{1}$, i.e., $\left|X_{i}^{1}\right| \times d_{u}$. We thus conclude that there is at least a set $X_{j}$ of Type 3.2 such that each node of $X_{i}$ has at least one neighbor in $X_{j}$, and by $\mathrm{P} 3,\left|X_{j}^{1}\right|>\left|X_{j}^{2}\right|$. But this contradicts (3). We thus come to the conclusion that this case cannot happen.

Case 2. Now, assume that $d_{w}=d_{u}$. If there are two nodes $y$ and $y^{\prime}$ in $Y$ such that $y$ is adjacent to $\frac{\left|X_{i}\right|}{2}$ nodes of $X_{i}^{1}$ and $y^{\prime}$ is adjacent to $\frac{\left|X_{i}\right|}{2}$ nodes of $X_{i}^{2}$, then $\frac{\left|X_{i}\right|}{2} \leq\left|X_{i}^{1}\right|$ and $\frac{\left|X_{i}\right|}{2} \leq\left|X_{i}^{2}\right|$. This implies $\left|X_{i}^{1}\right|=\left|X_{i}^{2}\right|=\frac{\left|X_{i}\right|}{2}$. But this contradicts the definition of Type 3.2 sets. Also, if there is a node $y \in Y$ such that $y$ is adjacent to $\frac{\left|X_{i}\right|}{2}$ nodes of $X_{i}^{1}$ (or $X_{i}^{2}$ ), but there is no node $y^{\prime} \in Y$ such that $y^{\prime}$ is adjacent to $\frac{\left|X_{i}\right|}{2}$ nodes of $X_{i}^{2}$ (or $X_{i}^{1}$ ), then by P5, we have $d_{u} \neq d_{w}$, again a contradiction. Thus, there is no connection between the nodes in $Y$ and those of $X_{i}$. Let $S$ be the union of all Type-3.2 sets. Partition $S$ into two sets $S^{1}$ and $S^{2}$, where $S^{1}$ is a subset of $U$ and $S^{2}$ is a subset of $W$. Each node in $S$ has no neighbor in $Y$, Type-1, Type-2, or Type-3.1 sets. Now, consider the node-induced subgraph on the set of nodes $S$. Since the degree of all nodes in $G$ are the same, by counting the number of edges from two sides, we have $\left|S^{1}\right|=\left|S^{2}\right|$. This combined with $d_{u}=d_{w}$, and Rule 3 shows that the Godsil-McKay switching does not change the degree sequence of $G$. The graph also remains bipartite. This completes the proof.

Remark 1. From the discussions above, one can see that a Godsil-McKay switching partition of bi-regular bipartite graphs, in which degrees of the two sides are unequal, cannot have Type 
3.2 sets. Thus, for practical Tanner graphs in which $d_{u} \neq d_{w}$, a valid Godsil-McKay switching partition $\left\{Y, X_{1}, \ldots, X_{\ell}\right\}$ of the nodes can only have $X_{i}$ 's that are either Type 1, Type 2 or Type 3.1. There can also be connections only between Types 1 and 2, and between Types 3.1 and 3.1. Nodes in $Y$ can be connected to the nodes in all three types of $X_{i}$ sets.

It is important to note that, in general, Godsil-McKay switching does not preserve the degree sequences of a graph. As an example, consider the half-regular bipartite graph $G$ shown in Fig. 4(a). Let $\ell=3$, and choose $X_{1}=\left\{v_{1}, v_{2}, v_{3}, v_{4}\right\}, X_{2}=\left\{u_{1}, u_{2}, u_{3}, u_{4}\right\}, X_{3}=\left\{z_{1}, z_{2}, z_{3}, z_{4}\right\}$ and $Y=\left\{x_{1}, x_{2}, x_{3}\right\}$. The partitioning has all necessary properties of Theorem 1, We can thus apply Godsil-McKay switching. By applying the switching, we obtain the graph $G^{\prime}$, given in Fig. 4(b). One can see that although $G^{\prime}$ is also half-regular with the same degree of two on the regular side, the degree sequence of the two graphs differ on the irregular side.

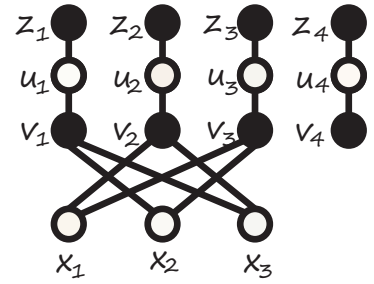

(a)

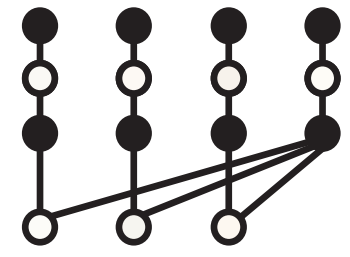

(b)

Fig. 4. Two cospectral graphs: (a) $G$ and (b) $G^{\prime}$.

\section{COMPUTING THE NUMBER OF CYCLES IN IRREGULAR BIPARTITE GRAPHS}

In this section, we consider the problem of counting the cycles of different length in irregular bipartite graphs of different girth $g$. First, we demonstrate through counter-examples that if $g=4$, the information of degree sequences and spectrum is, in general, insufficient to count $i$-cycles for any $i \geq g+2$. Next, for $g \geq 6$, we show by counter-examples that spectrum and degree sequences cannot, in general, uniquely determine the multiplicity of $i$-cycles for any $i \geq g$. The results for the case of $g \geq 8$ are provided before those of $g=6$, since the graphs constructed for the former case are used as building blocks for graph constructions in the latter. 
A. $g=4$ : Counter-example for $i$-cycles, $i \geq g+2$

In this subsection, we construct two half-regular bipartite graphs such that they both have the same spectrum, degree sequence and girth 4 , but have different number of $i$-cycles for $i \geq 6$.

Construction of the graph $\mathcal{G}$ :

Consider two disjoint cycles of length 4 and 12 with node sets $\left\{v_{1}, v_{2}, v_{3}, v_{4}\right\}$, and $\left\{u_{1}, u_{2}, \ldots, u_{12}\right\}$, respectively. Add two nodes $w$ and $w^{\prime}$ to the union of the cycles, and connect both $w$ and $w^{\prime}$ to the nodes $v_{1}, v_{3}, u_{1}, u_{3}$. Also, add another node $w^{\prime \prime}$, and connect it to the nodes $u_{5}, u_{7}, u_{9}, u_{11}$. Finally, add two more nodes $x$ and $y$ to the graph, and connect them to the nodes $v_{2}, v_{4}, u_{2}, u_{4}, u_{6}, u_{8}, u_{10}, u_{12}$. Call the resultant graph $\mathcal{G}$. The graph $\mathcal{G}$ is bipartite, and has 21 nodes and its girth is 4 . See Fig. 5 , Consider the node partition $V(G)=U \cup W$ for $\mathcal{G}$, where $W=\left\{x, y, v_{1}, v_{3}, u_{1}, u_{3}, u_{5}, u_{7}, u_{9}, u_{11}\right\}$. We thus have $n=|U|=11$ and $m=|W|=10$. The degree sequence of $W$ is $(8,8,4,4,4,4,3,3,3,3)$, and the degree of each node in $U$ is 4 . Thus, $\mathcal{G}$ is variable-regular with variable degree 4 .

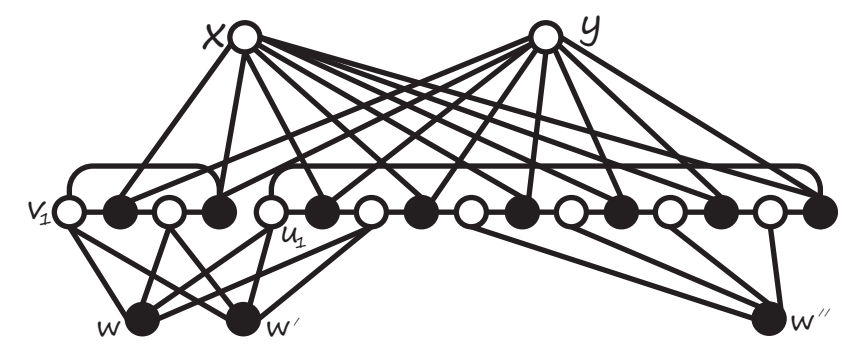

Fig. 5. Graph $\mathcal{G}$ of Subsection IV-A

Construction of $\mathcal{G}^{\prime}$ from $\mathcal{G}$ : We use Godsil-McKay switching of Theorem 1, We choose $\ell=2$, $X_{1}=\left\{v_{i}, u_{i}: i\right.$ is odd $\}$ and $X_{2}=\left\{v_{i}, u_{i}: i\right.$ is even $\}$. Thus, $\left|X_{1}\right|=\left|X_{2}\right|=8$. Also, we select $Y=\left\{w, w^{\prime}, w^{\prime \prime}, x, y\right\}$. Nodes $w, w^{\prime}$ and $w^{\prime \prime}$, each has 4 neighbors in $X_{1}$, and no neighbor in $X_{2}$. Also, each of the nodes $x$ and $y$ has 8 neighbors in $X_{2}$ and no neighbor in $X_{1}$. Moreover, for each pair $i, j \in\{1,2\}$, all nodes in $X_{i}$ have the same number of neighbors in $X_{j}$. The partitioning has all necessary properties of Theorem 1, and thus, we can apply Godsil-McKay switching. By applying the switching, we obtain the graph $\mathcal{G}^{\prime}$, which has the same degree sequences as $\mathcal{G}$. In Table IV, we have listed the cycle distribution of both graphs for cycle lengths up to 18. One can see that $\mathcal{G}$ and $\mathcal{G}^{\prime}$, although having the same spectrum, degree sequences and $g=4$, have different number of $i$-cycles for $i=6,8, \ldots, 18$. 
TABLE IV

Multiplicities of CyCles of Length 4 up to 18 In $\mathcal{G}$ And $\mathcal{G}^{\prime}$, CONSTRUCTEd in SubsectionIV-A

\begin{tabular}{|c||c|c|c|c|c|c|c|c|}
\hline Graph & 4-cycles & 6-cycles & 8-cycles & 10-cycles & 12-cycles & 14-cycles & 16-cycles & 18-cycles \\
\hline $\mathcal{G}$ & 60 & 248 & 1300 & 4056 & 11992 & 29780 & 43040 & 32640 \\
\hline $\mathcal{G}^{\prime}$ & 60 & 250 & 1294 & 4026 & 11706 & 28440 & 41656 & 32096 \\
\hline
\end{tabular}

\section{B. $g \geq 8$ : Counter-examples for $i$-cycles with $i \geq g$}

In this subsection, we consider irregular bipartite graphs with girth $g$ at least eight, and demonstrate that the information of spectrum, and degree sequences is not sufficient, in general, to determine the multiplicity of $i$-cycles for $i \geq g$. For this, in the following, for each $t \geq 1$, we first construct two irregular bipartite graphs $G_{t}$ and $G_{t}^{\prime}$ such that they have the same spectrum and degree sequences, but different number of $(6+2 t)$-cycles (one vs. zero). The disjoint union of these graphs can then be used to provide counter-examples for cospectral irregular graphs with the same degree sequences and the same girth $g$ (for any girth $g \geq 8$ ), but with different number of $i$-cycles for any $i \geq g$. (We note that the irregular graph constructed by the disjoint union of $G_{t}$ graphs, $t \geq \tau$, has girth $6+2 \tau$, while the corresponding disjoint union of $G_{t}^{\prime}$ graphs has an infinite girth. To make an example where both graphs have the same girth, one can simply consider the union of the constructed graphs with a cycle of length $6+2 \tau$.)

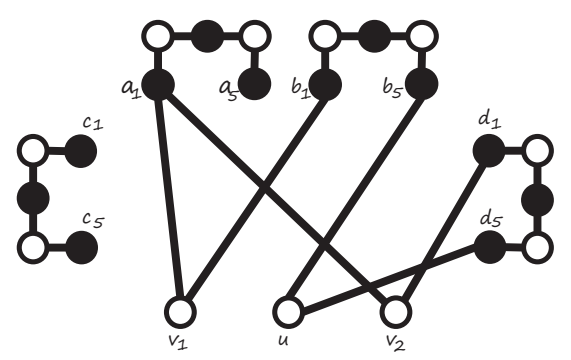

Fig. 6. Graph $G_{4}$ constructed in Subsection IV-B

Construction of the graph $G_{t}$ : Consider the integer $t \geq 1$, and four paths, each of length $t$, with the node sets $\left\{a_{1}, \ldots, a_{t+1}\right\},\left\{b_{1}, \ldots, b_{t+1}\right\},\left\{c_{1}, \ldots, c_{t+1}\right\}$, and $\left\{d_{1}, \ldots, d_{t+1}\right\}$, respectively. Then, add three nodes $v_{1}, v_{2}$ and $u$, and the edges $v_{1} a_{1}, v_{1} b_{1}, v_{2} a_{1}, v_{2} d_{1}, u d_{t+1}$ and $u b_{t+1}$, to the graph. Call the resultant bipartite graph $G_{t}$. As an example, the graph $G_{4}$ is shown in Fig. 6 , 
The graph $G_{t}$ has $4 t+7$ nodes and only one cycle of length $6+2 t$. From $4 t+7$ nodes, $4 t+3$ are of degree 2 , one node has degree 3 and three nodes have degree 1.

Constructing $G_{t}^{\prime}$ from $G_{t}$ : We use Godsil-McKay switching of Theorem 1 with $\ell=t+1$, and for each $i, 1 \leq i \leq t+1$, we select $X_{i}=\left\{a_{i}, b_{i}, c_{i}, d_{i}\right\}$. We thus have $Y=\left\{v_{1}, v_{2}, u\right\}$. It can be seen that, for every node $y \in Y$, and every $i \in\{1, \ldots, t+1\}$, the node $y$ has either 0 or 2 neighbors in $X_{i}$ (note that for each $i,\left|X_{i}\right|=4$ ). Also, for each pair $i, j \in\{1, \ldots, t+1\}$, all the nodes in $X_{i}$ have the same number of neighbors in $X_{j}$. The $(i, j)$ entry of the following matrix shows the number of neighbors that an arbitrary node $v \in X_{i}$ has in the set $X_{j}$ :

$$
\left[\begin{array}{cccccccc}
0 & 1 & 0 & 0 & \cdots & 0 & 0 & 0 \\
1 & 0 & 1 & 0 & \cdots & 0 & 0 & 0 \\
0 & 1 & 0 & 1 & \cdots & 0 & 0 & 0 \\
\vdots & \vdots & \vdots & \vdots & \ddots & \vdots & \vdots & \vdots \\
0 & 0 & 0 & 0 & \cdots & 1 & 0 & 1 \\
0 & 0 & 0 & 0 & \cdots & 0 & 1 & 0
\end{array}\right] .
$$

This matrix shows that for each pair $i, j \in\{1, \ldots, \ell\}$, all the nodes in $X_{i}$ have the same number of neighbors in $X_{j}$. Thus, the node partitioning has all the necessary properties of Theorem 1 for the application of Godsil-McKay switching. By applying the switching, we obtain the graph $G_{t}^{\prime}$. For example, corresponding to $G_{4}$ in Fig. 6, we obtain the graph $G_{4}^{\prime}$, shown in Fig. 7, The graph $G_{t}^{\prime}$ has the same spectrum and degree sequences as $G_{t}$, but does not have any cycle.

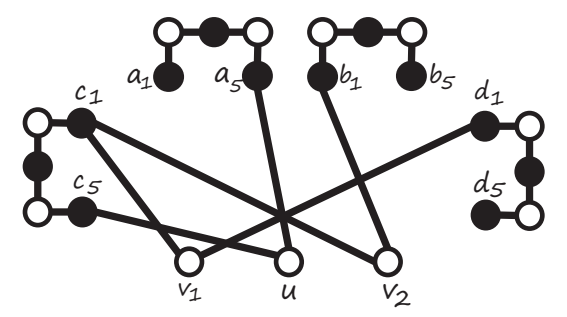

Fig. 7. Graph $G_{4}^{\prime}$, obtained by Godsil-McKay switching from $G_{4}$, shown in Fig. 6

Remark 2. Consider the graph $G_{t}$, where $t$ is an even number. Consider the partition $V\left(G_{t}\right)=$ $U \cup W$ for the nodes of $G_{t}$, where $U=\left\{v_{1}, v_{2}, u\right\} \cup\left\{a_{i}, b_{i}, c_{i}, d_{i}: i\right.$ is even $\}$. The graphs $G_{t}$ and $G_{t}^{\prime}$ are variable-regular with variable degree 2. We thus conclude that the number of $g$-cycles in half-regular bipartite graphs cannot be, in general, computed using the spectrum and the degree 
sequences of the graph when the girth of the variable-regular bipartite graph is $g=6+2 t$, where $t \geq 2$ is an even number.

C. $g=6$ : Counter-examples for $i$-cycles with $i \geq g$

In this subsection, we first provide a counter-example of two cospectral irregular graphs with similar degree sequences and $g=6$, but different $N_{6}$. In Part IV-C2, we then construct two irregular bipartite graphs $G_{t, k}$ and $G_{t, k}^{\prime}$ with girth 6 , such that they both have the same spectrum and degree sequences, but different multiplicity for $i$-cycles with $i \geq g+2$.

1) Counter-example for 6-cycles: Consider the disjoint union of two 6-cycles and two paths, each of length 5 , and call it $G_{1}$ (i.e., $G_{1}=2 C_{6} \cup 2 P_{6}$ ). Also, consider the disjoint union of a 6-cycle, a 14-cycle and two paths, each of length one, and call it $G_{2}$ (i.e., $G_{2}=C_{6} \cup C_{14} \cup 2 P_{2}$ ). It is easy to see that $G_{1}$ and $G_{2}$ are irregular bipartite graphs with the same degree sequences (both have ten nodes with degree 2 and two nodes with degree 1 on each side of the bipartition). Using (1) and (2), one can also see that $G_{1}$ and $G_{2}$ are cospectral. The girth of both graphs is six, but they have different number of 6-cycles (two vs. one).

2) Counter-example for $i$-cycles with $i \geq g+2$ : Construction of the graph $G_{t, k}$ : Let $t$ and $k$ be two integers such that $t>k \geq 0$, and $t+k$ is an even number. Consider the graph $G_{t}$ which was constructed in Subsection IV-B. Add a path of length $k$ with the node set $\left\{f_{1}, \ldots, f_{k+1}\right\}$, as well as the edges $u f_{1}$ and $v_{2} f_{k+1}$ to $G_{t}$. Call the resultant graph $G_{t, k}$. As an example, the graph $G_{4,2}$ is shown in Fig. 8 (a). The graph $G_{t, k}$ has $4 t+k+8$ nodes, out of which, $4 t+k+2$ nodes have degree 2 , three have degree 3 , and three have degree 1 . The graph is also bipartite and has one cycle of length $t+k+4$, one cycle of length $t+k+6$ and one cycle of length $6+2 t$.

Construction of $G_{t, k}^{\prime}$ from $G_{t, k}$ : We use Godsil-McKay switching to transform $G_{t, k}$ into $G_{t, k}^{\prime}$. Let $\ell=t+1$, and for each $i, 1 \leq i \leq t+1$, let $X_{i}=\left\{a_{i}, b_{i}, c_{i}, d_{i}\right\}$. We then have $Y=\left\{v_{1}, v_{2}, u, f_{1}, f_{2}, \ldots, f_{k+1}\right\}$. All the conditions of Theorem 1 apply to this partition. We call the graph obtained by the switching $G_{t, k}^{\prime}$. The graph $G_{t, k}^{\prime}$ can also be generated directly from $G_{t}^{\prime}$, the Godsil-McKay switched version of $G_{t}$ : Add to $G_{t}^{\prime}$ a path of length $k$ with the node set $\left\{f_{1}, \ldots, f_{k+1}\right\}$, and the edges $u f_{1}$ and $v_{2} f_{k+1}$. As an example, in Fig. 8(b), the graph $G_{4,2}^{\prime}$ is shown. The graph $G_{t, k}^{\prime}$ has the same spectrum and degree sequences as $G_{t, k}$, but has only one cycle of length $t+k+4$. Now, for a fixed $i \geq 1$, consider the disjoint union of graphs $G_{2,0}, G_{3,1}, \ldots, G_{i+2, i}$, and call it $D_{i}$. Also, use $D_{i}^{\prime}$ to denote the disjoint union of graphs 


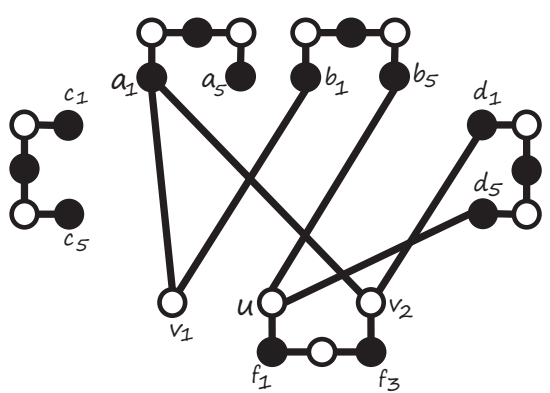

(a)

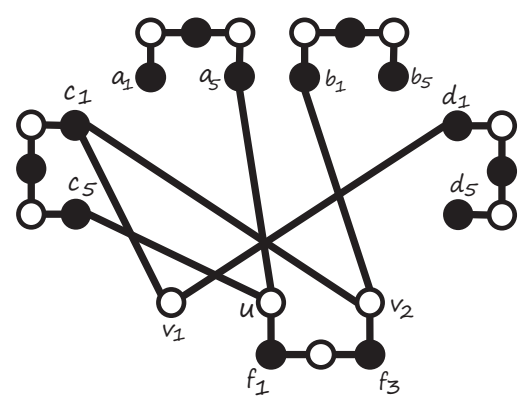

(b)

Fig. 8. Graphs (a) $G_{4,2}$ and (b) $G_{4,2}^{\prime}$.

$G_{2,0}^{\prime}, G_{3,1}^{\prime}, \ldots, G_{i+2, i}^{\prime}$. For each $j \in\{0, \ldots, i\}$, the graph $G_{j+2, j}$ has one $(2 j+6)$-cycle, one $(2 j+8)$-cycle and one $(2 j+10)$-cycle. Also, the graph $G_{j+2, j}^{\prime}$ has only one cycle of length $2 j+6$. Considering that the spectrum of a disconnected graph is the disjoint union of the spectra of its components, one can see that $D_{i}$ and $D_{i}^{\prime}$ are cospectral. They also have the same degree sequences and girth $g=6$. It can however, be seen that while both graphs have only one cycle of length 6 , they have different number of $k$-cycles for each $6<k \leq 2 i+10$. As an example, the cycle distributions of $D_{3}$ and $D_{3}^{\prime}$ are given in Table $\mathrm{V}$.

TABLE V

Multiplicities of CyCles of LENGTH 6 UP to 16 in GRAPHS $D_{3}$ AND $D_{3}^{\prime}$

\begin{tabular}{|c||c|c|c|c|c|c|}
\hline Graph & 6-cycles & 8-cycles & 10-cycles & 12-cycles & 14-cycles & 16-cycles \\
\hline$D_{3}$ & 1 & 2 & 3 & 3 & 2 & 1 \\
\hline$D_{3}^{\prime}$ & 1 & 1 & 1 & 1 & 0 & 0 \\
\hline
\end{tabular}

\section{COUNTING CYCLES IN HALF-REGULAR BIPARTITE GRAPHS}

The counter-example constructed in Subsection IV-A for $g=4$ was based on half-regular bipartite graphs. We thus know that if $g=4$, the knowledge of spectrum and degree sequences is not sufficient in general to count the number of $i$-cycles for $i \geq g+2$ in half-regular bipartite graphs. On the other hand, the positive result of [7] is applicable to half-regular graphs and can be used to compute $N_{4}$. Furthermore, in Remark 2, we showed that, in general, one cannot find $N_{g}$ for $g=6+2 t$, where $t \geq 2$ is an even number, in half-regular graphs just by using the 
information of spectrum and degree sequences. In this section, we complement these results. We present counter-examples for $g$-cycles if $g=6+2 t$, where $t \geq 1$ is an odd number, or for $i$-cycles with $i \geq g+2$, in graphs with $g \geq 6$.

\section{A. Counter-examples for $g$-cycles $(g=6+2 t, t \geq 1$ and odd $)$}

Consider the disjoint union of two cycles, each of length $6+2 t$, and two paths, each of length $5+t$, and call it $G_{1}$. Also, consider the disjoint union of a $(6+2 t)$-cycle, a $(14+2 t)$-cycle and two paths, each of length $t+1$, and call it $G_{2}$. One can see that both $G_{1}$ and $G_{2}$ are half-regular bipartite graphs and have the same degree sequences (the regular side has $11+3 t$ degree- 2 nodes and the irregular side has $9+3 t$ degree-2 and 4 degree- 1 nodes) 2 Using (1) and (2), one can also see that $G_{1}$ and $G_{2}$ are cospectral, and both have girth $g=6+2 t$. The number of $g$-cycles $N_{g}$, however, is different for each graph (two vs. one).

\section{B. $g \geq 6$ : Counter-examples for $i$-cycles, $i \geq g+2$}

In this subsection, we construct variable-regular bipartite graphs that have the same spectrum, degree sequences and girth $g \geq 6$, but have different multiplicities of $i$-cycles for $i \geq g+2$. We first start by constructing two graphs $\mathcal{G}_{t, k}$ and $\mathcal{G}_{t, k}^{\prime}$, related by Godsil-McKay switching.

Construction of the graph $\mathcal{G}_{t, k}$ : Let $t$ and $k$ be two even integers such that $t \geq k \geq 0$ and $t>0$. Consider the graph $G_{t, k}$ which was constructed in Subsection IV-C. For each node $z$ in the set $\left\{a_{i}, b_{i}, c_{i}, d_{i}, f_{i}: i\right.$ even $\} \cup\left\{v_{1}\right\}$, add a new node $z^{\prime}$ to $G_{t, k}$, and connect $z$ to $z^{\prime}$. Call the resultant variable-regular graph $\mathcal{G}_{t, k}$. As an example, Fig. 9 (a) shows $\mathcal{G}_{4,2}$. The graph $\mathcal{G}_{t, k}$ is bipartite and has one cycle of length $t+k+4$, one cycle of length $t+k+6$ and one cycle of length $6+2 t$. (Note that if $t=k$, then the graph $\mathcal{G}_{t, k}$ has one cycle of length $2 t+4$, and two cycles of length $2 t+6$.)

Construction of the graph $\mathcal{G}_{t, k}^{\prime}$ : We use Godsil-McKay switching of Theorem 1 to construct $\mathcal{G}_{t, k}^{\prime}$ from $\mathcal{G}_{t, k}$. Let $\ell=3 t / 2+1$, and for each $i, 1 \leq i \leq t+1$, let $X_{i}=\left\{a_{i}, b_{i}, c_{i}, d_{i}\right\}$. Also, for each $i, t+2 \leq i \leq 3 t / 2+1$, let $j=2(i-t-1)$, and $X_{i}=\left\{a_{j}^{\prime}, b_{j}^{\prime}, c_{j}^{\prime}, d_{j}^{\prime}\right\}$. We thus have $Y=\left\{v_{1}, v_{2}, u, f_{1}, f_{2}, \ldots, f_{k+1}\right\} \cup\left\{f_{i}^{\prime}: i\right.$ even $\} \cup\left\{v_{1}^{\prime}\right\}$. It can be seen that this partitioning satisfies all the required conditions of Theorem 1 . We thus apply the switching and obtain the graph $\mathcal{G}_{t, k}^{\prime}$. The graph $\mathcal{G}_{t, k}^{\prime}$ can also be constructed by the following approach: Consider the graph $G_{t, k}^{\prime}$ which

\footnotetext{
${ }^{2}$ Note that if $t$ is selected to be an even number, the graphs $G_{1}$ and $G_{2}$ will not be half-regular.
} 
was constructed in Subsection IV-B. For each node $z$ in the set $\left\{a_{i}, b_{i}, c_{i}, d_{i}, f_{i}: i\right.$ even $\} \cup\left\{v_{1}\right\}$, add a new node $z^{\prime}$ to $G_{t, k}^{\prime}$, and connect $z$ to $z^{\prime}$. As an example, Fig. 9(b) shows the graph $\mathcal{G}_{4,2}^{\prime}$. The graph $\mathcal{G}_{t, k}^{\prime}$ is also variable-regular bipartite and has the same spectrum and degree sequences as $\mathcal{G}_{t, k}$. It however, has only one cycle of length $t+k+4$.

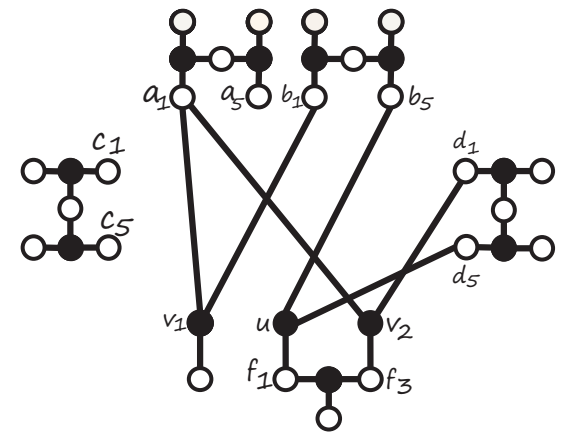

(a)

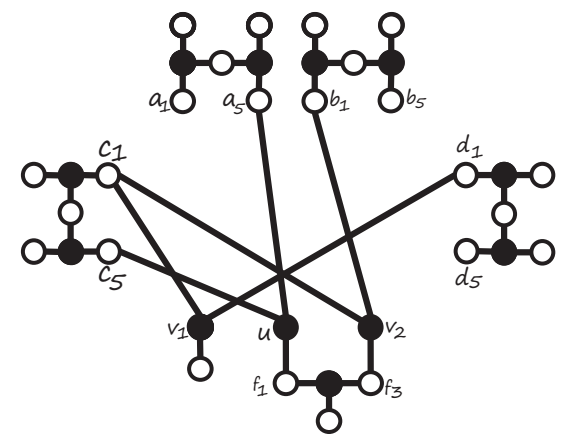

(b)

Fig. 9. Graphs (a) $\mathcal{G}_{4,2}$ and (b) $\mathcal{G}_{4,2}^{\prime}$.

Let $i$ be an even number. The graph $\mathcal{G}_{i+2, i}$ has one $(2 i+6)$-cycle, one $(2 i+8)$-cycle and one $(2 i+10)$-cycle. The graph $\mathcal{G}_{i+2, i}^{\prime}$, however, has only one cycle of length $2 i+6$. Now, for fixed integers $j$ and $k$ satisfying $j \geq k \geq 1$, consider the disjoint union of graphs $\mathcal{G}_{2 k, 2 k-2}, \mathcal{G}_{2 k+2,2 k}, \ldots, \mathcal{G}_{2 j, 2 j-2}$, and call it $\mathcal{F}_{j, k}$. Also, consider the disjoint union of graphs $\mathcal{G}_{2 k, 2 k-2}^{\prime}, \mathcal{G}_{2 k+2,2 k}^{\prime}, \ldots, \mathcal{G}_{2 j, 2 j-2}^{\prime}$, and call it $\mathcal{F}_{j, k}^{\prime}$. Both $\mathcal{F}_{j, k}$ and $\mathcal{F}_{j, k}^{\prime}$ have the same spectrum and degree sequences. They also have the same girth of $4 k+2$, and both have one $(4 k+2)$-cycle. They however, have different number of $\ell$-cycles for any $4 k+2<\ell \leq 4 j+6$.

To cover the cases where $g=4(k+1), k \geq 1$, let $k^{\prime}$ be an odd number satisfying $k^{\prime}>2 k+1$, and consider two graphs $G_{1}$ and $G_{2}$, where $G_{1}$ is the disjoint union of the cycle $C_{4(k+1)}$ and two copies of the path $P_{k^{\prime}}$, and $G_{2}$ is the disjoint union of $C_{2\left(k^{\prime}+1\right)}$, and two copies of the path $P_{2 k+1}$. One can see that $G_{1}$ and $G_{2}$ are half-regular bipartite graphs with similar degree sequences. It can also be seen, using (1) and (2), that both graphs have the same spectrum. The two graphs, however, have different cycle distributions, i.e., while $G_{1}$ has one cycle of length $4(k+1), G_{2}$ has one cycle of larger length $2\left(k^{\prime}+1\right)$. Now, if one considers the disjoint unions of $G_{1}$ and $G_{2}$ with a cycle of length $4(k+1)$, then the resultant graphs both have the same girth of $4(k+1)$, but they have different number of cycles of length $2\left(k^{\prime}+1\right)$. 


\section{CONCLUDING REMARKS}

It is well-known that the number of closed walks in a graph can be computed using the spectrum of the graph. It is also known that the multiplicity of cycles of length larger than three cannot be determined only by the knowledge of the spectrum. Recently, in [2], [7], it was shown that adding the knowledge of degree sequences for bipartite graphs to the information about the spectrum will enable the computation of multiplicities of cycles of certain lengths in bi-regular, half-regular and irregular graphs. (See Table I) In this work, we complemented the results of [2], [7], and demonstrated, by constructing counter-examples, that for the remaining cases, the information of the spectrum and degree sequences is insufficient, in general, to determine the multiplicity of cycles. An interesting topic of research would be to determine what extra information, in addition to degree sequences and spectrum, is required to compute the multiplicity of cycles of length larger than or equal to $2 g$.

In Theorem 2, we proved that Godsil-McKay switching preserves the degree sequences of bi-regular bipartite graphs. It is also known that the spectrum of the graph is preserved under this switching. This implies that the multiplicity of short cycles of length up to $2 g-2$ remains unchanged with the application of the Godsil-McKay switching to a bi-regular bipartite graph. On the other hand, short cycles and their combinations form graphical objects that trap the iterative decoding algorithms of LDPC codes. An interesting topic would be to study the effect of Godsil-McKay switchings on the distribution of trapping sets and the possibility of reducing the multiplicity of trapping sets through the application of this switching.

\section{REFERENCES}

[1] R. Asvadi, A. H. Banihashemi, and M. Ahmadian-Attari. Lowering the error floor of LDPC codes using cyclic liftings. IEEE Trans. Inform. Theory, 57(4):2213-2224, 2011.

[2] I. F. Blake and S. Lin. On short cycle enumeration in biregular bipartite graphs. IEEE Trans. Inform. Theory, 64(10):65266535, 2018.

[3] Z. L. Blázsik, J. Cummings, and W. H. Haemers. Cospectral regular graphs with and without a perfect matching. Discrete Math., 338(3):199-201, 2015.

[4] J. A. Bondy and U. S. R. Murty. Graph theory with applications. American Elsevier Publishing Co., Inc., New York, 1976.

[5] A. E. Brouwer and W. H. Haemers. Eigenvalues and perfect matchings. Linear Algebra Appl., 395:155-162, 2005.

[6] A. Dehghan and A. H. Banihashemi. On the Tanner graph cycle distribution of random LDPC, random protograph-based LDPC, and random quasi-cyclic LDPC code ensembles. IEEE Trans. Inform. Theory, 64(6):4438-4451, 2018. 
[7] A. Dehghan and A. H. Banihashemi. On computing the multiplicity of cycles in bipartite graphs using the degree distribution and the spectrum of the graph. accepted to IEEE Trans. Inform. Theory, Dec. 2018, available online at: https://arxiv.org/abs/1806.01433.

[8] R. G. Gallager. Low-Density Parity-Check Codes. Cambridge, MA: MIT Press, 1963.

[9] C. D. Godsil and B. D. McKay. Constructing cospectral graphs. Aequationes Math., 25(2-3):257-268, 1982.

[10] T. R. Halford and K. M. Chugg. An algorithm for counting short cycles in bipartite graphs. IEEE Trans. Inform. Theory, 52(1):287-292, 2006.

[11] Y. Hashemi and A. H. Banihashemi. New characterization and efficient exhaustive search algorithm for leafless elementary trapping sets of variable-regular LDPC codes. IEEE Trans. Inform. Theory, 62(12):6713-6736, 2016.

[12] Y. Hashemi and A. H. Banihashemi. Characterization of elementary trapping sets in irregular LDPC codes and the corresponding efficient exhaustive search algorithms. IEEE Trans. Inform. Theory, 64(5):3411-3430, 2018.

[13] J. Howbert. Count all cycles in simple undirected graph, version 1.2, URL: https://www.mathworks.com/matlabcentral/fileexchange/29438-count-all-cycles-in-simple-undirected-graph.

[14] X.-Y. Hu, E. Eleftheriou, and D. M. Arnold. Regular and irregular progressive edge-growth Tanner graphs. IEEE Trans. Inform. Theory, 51(1):386-398, 2005.

[15] A. M. W. Hui and B. G. Rodrigues. Switched graphs of some strongly regular graphs related to the symplectic graph. Des. Codes Cryptogr., 86(1):179-194, 2018.

[16] M. Karimi and A. H. Banihashemi. Efficient algorithm for finding dominant trapping sets of LDPC codes. IEEE Trans. Inform. Theory, 58(11):6942-6958, 2012.

[17] M. Karimi and A. H. Banihashemi. On the girth of quasi-cyclic protograph LDPC codes. IEEE Trans. Inform. Theory, 59(7):4542-4552, 2013.

[18] M. Karimi and A. H. Banihashemi. On characterization of elementary trapping sets of variable-regular LDPC codes. IEEE Trans. Inform. Theory, 60(9):5188-5203, 2014.

[19] M. Karimi and A. H. Banihashemi. Message-passing algorithms for counting short cycles in a graph. IEEE Trans. Communications, 61(2):485-495, Feb. 2013.

[20] F. R. Kschischang, B. J. Frey, and H.-A. Loeliger. Factor graphs and the sum-product algorithm. IEEE Trans. Inform. Theory, 47(2):498-519, 2001.

[21] M. Langberg and D. Vilenchik. Constructing cospectral graphs via a new form of graph product. Linear Multilinear Algebra, 66(9):1838-1852, 2018.

[22] M. Liu, Y. Yuan, L. You, and Z. Chen. Which cospectral graphs have same degree sequences. Discrete Math., 341(11):29692976, 2018.

[23] H. Lu. Regular graphs, eigenvalues and regular factors. J. Graph Theory, 69(4):349-355, 2012.

[24] H. Müller. Hamiltonian circuits in chordal bipartite graphs. Discrete Math., 156(1-3):291-298, 1996.

[25] A. Munemasa. Godsil-McKay switching and twisted Grassmann graphs. Des. Codes Cryptogr., 84(1-2):173-179, 2017.

[26] G. R. Omidi. On integral graphs with few cycles. Graphs Combin., 25(6):841-849, 2009.

[27] T. J. Richardson and R. L. Urbanke. The capacity of low-density parity-check codes under message-passing decoding. IEEE Trans. Inform. Theory, 47(2):599-618, 2001.

[28] H. Saeedi and A. H. Banihashemi. New sequences of capacity achieving LDPC code ensembles over the binary erasure channel. IEEE Trans. Inform. Theory, 56(12):6332-6346, 2010.

[29] D. Stevanović. Two spectral characterizations of regular, bipartite graphs with five eigenvalues. Linear Algebra Appl., 435(10):2612-2625, 2011.

[30] R. M. Tanner. A recursive approach to low complexity codes. IEEE Trans. Inform. Theory, 27(5):533-547, 1981. 
[31] E. R. van Dam and W. H. Haemers. Which graphs are determined by their spectrum? Linear Algebra Appl., 373:241-272, 2003.

[32] H. Xiao and A. H Banihashemi. Error rate estimation of low-density parity-check codes on binary symmetric channels using cycle enumeration. IEEE Trans. Commun., 57(6), 2009. 